\title{
Analysis on vascular bifurcation Y-type coronary artery based on hemodynamic
}

\author{
Ye $\mathrm{Lu}^{1, \text { a }}$, Huayong $\mathrm{Wu}^{2, \mathrm{~b}^{*}}$ \\ ${ }^{1}$ Jingshi Road 17923, Jinan, China \\ ${ }^{2}$ Haitang Road 5001, Jinan, China \\ aluye19860706@163.com \\ bshshzhang@sdu.edu.cn
}

Keywords: Y-type coronary artery, thrombus, hemodynamic

Abstract. This article analyses the fluid dynamic characters of bloodstream in Y-type coronary artery based on fluid dynamics. Velocity at the inlet is given for boundary condition. The speed is equally distributed. The fluid conditions of bloodstream at bifurcation and in branch vessels are analysed in the main text. The velocity, pressure, shear stress and energy loss are studied in this process. The results show that the vascular bifurcation is the optimal location for thrombus due to the low speed and high pressure.

\section{Introduction}

Thrombus may block vascular, causing less blood supplies in human body. Once the unstable thrombus falls off, it may remove with bloodstream to the other organs and cause organ functional disease. According to statistical material, the number of deaths caused by vascular disease takes a portion of $51 \%$ of total deaths caused by disease. It has become a main threats to human health.

According to research, thrombus mainly happens at the location of vascular bifurcation and vascular bend. High speed and vortex are most likely to happen. [1]So the study of fluid characters is meaningful both for theory development and in practical value.

The current studies on thrombus focus on clinic treatment[2], endovascular stent[3,4] and theombolitic treatment[5]. This article studies the velocity and mechanical properties of bloodstream passing through vascular bifurcation of Y-type coronary artery based on fluid dynamics and hemodynamic.

\section{The model of vascular bifurcation of Y-type coronary artery}

Vascular bifurcations of coronary artery are normally classified by two different types, Y-type and T-type. This article has built up a geometric model of Y-type vessel bifurcation of which the branch angle is less than 70 degree. The model is built as shown in figure 1.

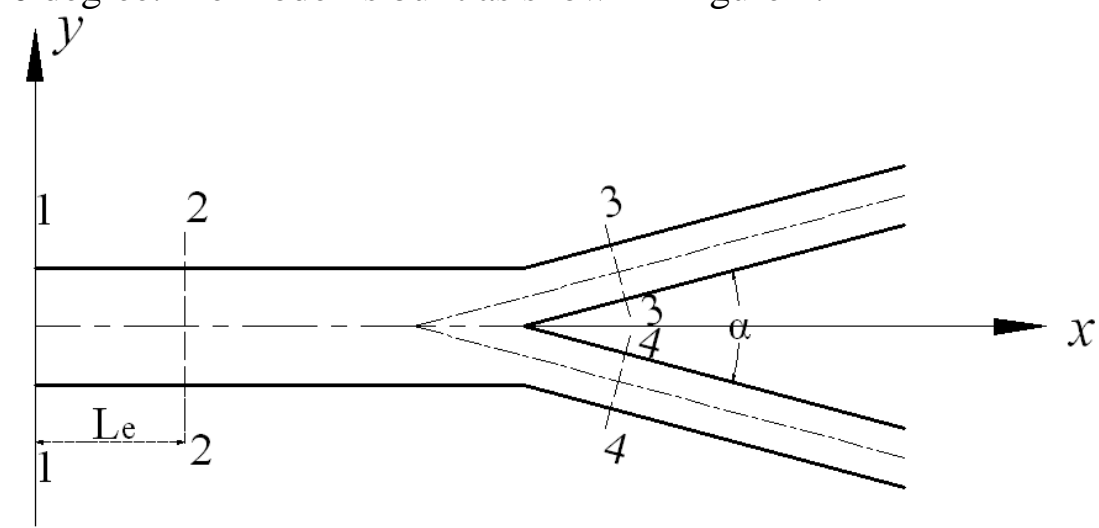

Figure 1. Model of Y-type vascular bifurcation of coronary artery

Due to the radial deformation of coronary artery is much smaller the diameter, also the flexibility of vessels will be reduced when vasculopathy occurs, thus the vessels of coronary artery can be 
equivalent to rigid circular pipe. Bloodstream is assumed incompressible in this article, and viscosity is constant.

The speed of bloodstream equally distributed at the entrance of vessel (section 1-1), the speed is $v_{1}$. The bloodstream is flowing in laminar motion in vessels. The radius of main vessel is $\mathrm{R}$, and length is $L_{1}$. The radius of two branch vessels has same radius defined as $R^{\prime}$, and be symmetrical about $\mathrm{X}$-axis. The vascular bifurcation of Y-type coronary artery is located horizontally. The gravity of blood stream is ignored. The pressure of bloodstream at the entrance is indicated as $p_{1}$.

\section{The analysis of the location of vascular bifurcation}

1. bloodstream at the location of vascular bifurcation

When bloodstream goes through the location of vascular bifurcation, the speed will be slow down due to the influence of vascular wall, then vortex and turbulent fluctuation will take place. Bloodstream will become a low-speed area in front of the vascular bifurcation. The pressure towards the vascular wall will increase in a short time because of the speed change. According to the momentum equation, the acting force against bloodstream which pass through the vascular bifurcation can be described as Eq. (1):

$$
\sum F=\frac{\Delta(m v)}{\Delta t}=-2 \rho q v_{1}\left(1-\frac{r^{2}}{R^{2}}\right)
$$

According to the relationship between acting force and reacting force, the acting force against vascular wall on bifurcation position is shown as Eq. (2):

$$
\sum F^{\prime}=2 \rho q v_{1}\left(1-\frac{r^{2}}{R^{2}}\right)
$$

The speed of bloodstream passing through the vascular bifurcation can be decomposed into 2 velocity components which are parallel to the axis of branch vessel and vertical to the axis of branch vessel, indicated as $v_{r}$ and $v_{n}$.

$$
\begin{aligned}
& v_{r}=2 v_{1}\left(1-\frac{r^{2}}{R^{2}}\right) \cos \frac{\theta}{2} \\
& v_{n}=2 v_{1}\left(1-\frac{r^{2}}{R^{2}}\right) \sin \frac{\theta}{2}
\end{aligned}
$$

There has been a significant change on the velocity component $v_{n}$ of the area nearby upper vascular wall of the lower branch vessel due to the acting force of vascular wall. So the wall shear stress will be observably here. The wall shear stress will become higher along with the increase of the branch angle. However, the wall shear stress will be lower along with the increase of the branch angle nearby the lower vascular wall of the lower branch.

According to the symmetry of the branch vessels, the conclusion for upper branch vessel can be drawing as well: the wall shear stress and pressure of lower wall will be significantly higher, while the wall shear stress and pressure of upper wall will be lower.

High pressure agaist vessel walls may cause injuries to vessel wlls.[6] Meanwhile the hemocyte and thrombocyte will accumulate at the location, so that it may become the optimal location for thrombogenesis.[7] 
2. the bloodstream in branch vessels

According to Murray' law, the relationship between the radiuses of branch vessel and main vessel can be described as: the cube of radius value of main vessel equals to the total summation of the cubes of radius value of each branch vessel. In this case, it can be revealed as Eq. (5):

$$
R^{3}=2 R^{3}
$$

When bloodstream flows in Y-type bifurcate coronary artery, the relationship of the speed of main vessel bloodstream $v_{2}$ and speed of one branch vessel bloodstream ${ }^{v_{3}}$ can be indicated as Eq. (6) :

$$
\iint_{2-2} \rho v_{2} d A=\iint_{3-3} \rho v_{3} d A+\iint_{4-4} \rho v_{3} d A
$$

According to Eq. (30) and Eq. (31), the fully-developed speed of bloodstream in branch vessels is shown in Eq. (7):

$$
v_{3}=2^{-\frac{1}{3}} v_{2}=2^{\frac{2}{3}} v_{1}\left(1-\frac{r^{2}}{R^{2}}\right)
$$

It is indicated that the speed will be lower after flowing into branch vessel, the speed of branch vessel is $2^{-1 / 3}$ times of the speed in main vessel. According to Newton Shear Theorem:

$$
\tau_{3}=\mu \frac{d v_{3}}{d r}=-\frac{2^{3 / 5} \mu v_{1}}{R^{2}} r
$$

The speed distribution is revealed in figure 2 down below:

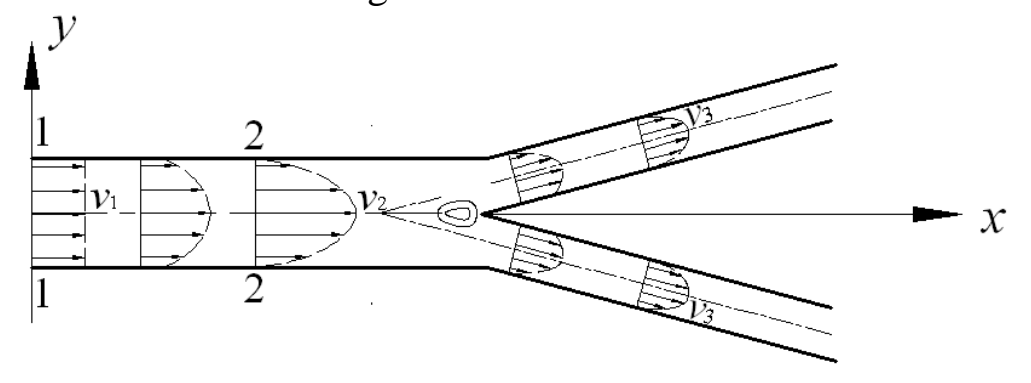

Figure 2. The speed distribution of Y-type bifurcate coronary artery

According to Bernoulli equation:

$$
h_{f 3}=\frac{p_{2}-p_{3}}{\rho}+\left(1-2^{-\frac{2}{3}}\right) v_{1}^{2}
$$

$p_{3}$ is referred to the intensity of pressure of the branch section, ${ }_{f 3}$ stands for the energy loss at the location of vascular bifurcation.

Eq. (9) reveals that the energy loss of vascular bifurcation consists pressure energy and kinetic energy. Due to the increasing loss of kinetic energy, the total energy loss at the vascular bifurcation is higher than other locations.

\section{Numerical simulation and analysis}

The numerical modeling is realized in Fluent. The parameter settings are obtained referring to the real blood parameter. However there is no unified value of blood parameters because it changes with time and different persons. So the value adopted in this article is the statistic value. 
The angle between two branch vessels is 50 degree. The branch vessels are symmetric about the main vessel axis. The radius of main vessel and branch vessels satisfied Eq. (30). The inlet velocity is $0.4 \mathrm{~m} / \mathrm{s}$. The viscosity of blood is $0.004 \mathrm{~kg} / \mathrm{ms}$. The density is $1055 \mathrm{~kg} / \mathrm{m}^{3}$.

The result of numerical simulation to the pressure and velocity is shown in figure 6 and figure 7 down below.

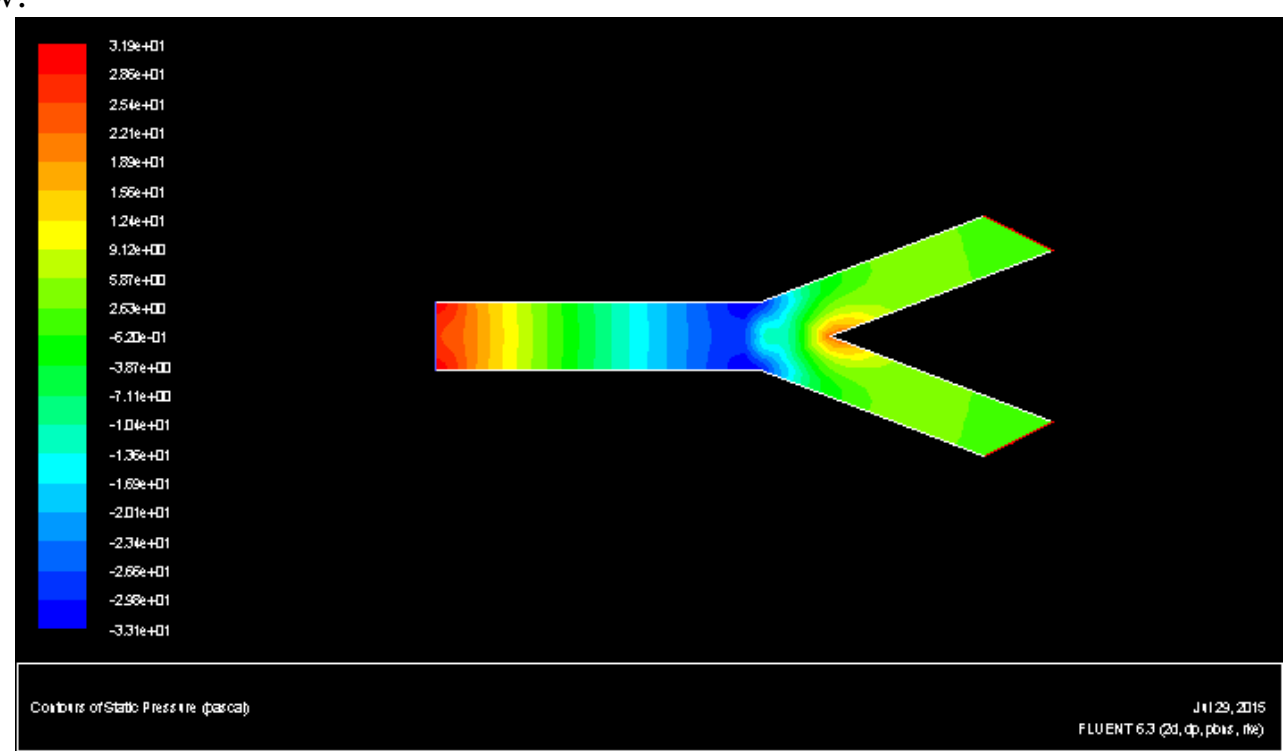

Figure 3. The pressure of vascular bifurcation

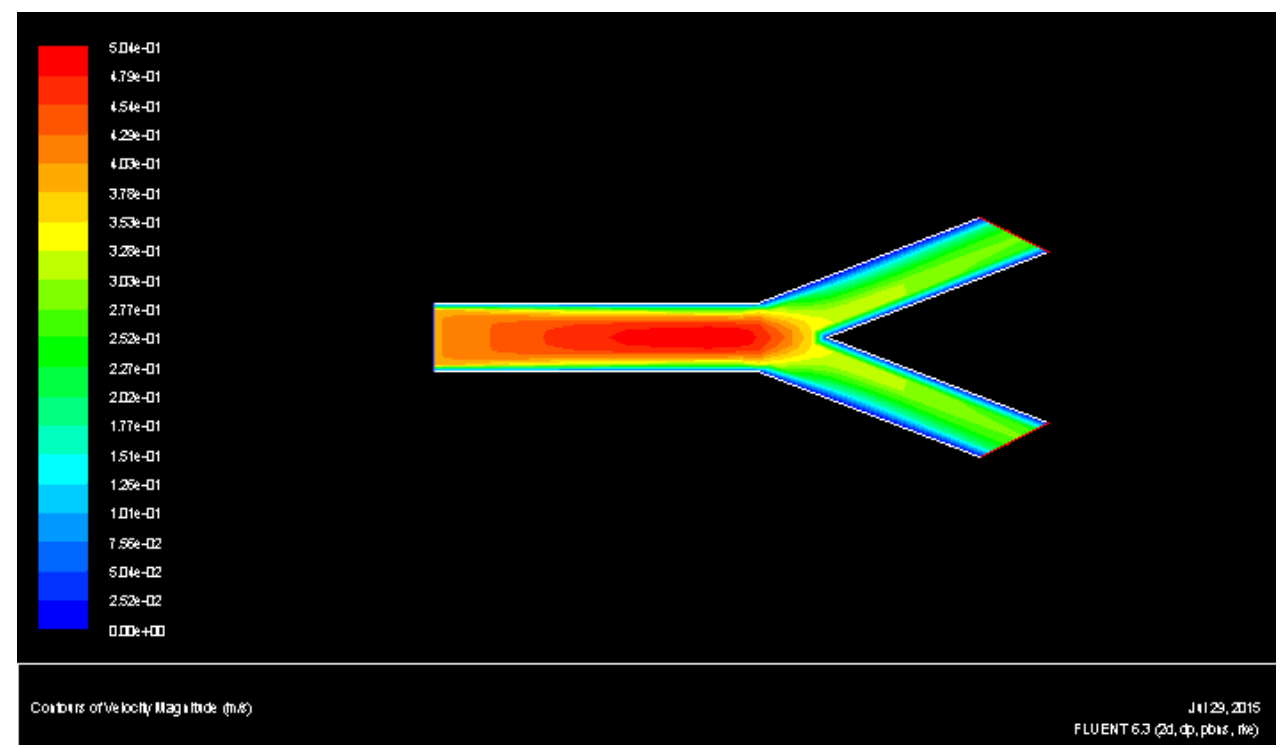

Figure 4. The velocity of vascular bifurcation

According to figure 6, pressure is much higher at the location of vascular bifurcation. So it is the location where thrombus most likely happened. The pressure becomes lower in branch vessels. So that the shear stress will decrease as well.

According to figure 7, an vortex appears in front of the vascular bifurcation. The particular matters may deposition in vortex. The vessel wall of vascular bifurcation can be damage by particular matters driven by shear stress. It shows that the optimal location for vasculopathy. The speed slows down in branch vessels.

\section{Conclusion}

Based on flow dynamics and hemodynamic, this article studied the speed, pressure, shear stress and energy loss in the process of blood flow. The conclusion can be as below: 
1. When bloodstream flows into main vessel, the speed near vessel wall slows down gradually, and the speed in center increases. Speed will retain constant as fully developed flow is formed. It is a stable value on the cross section of through-flow. The speed is in parabolic distribution.

2. The shear stress is in linear distribution as the fully developed flow is formed.

3. Vortex will take place in at the location of vascular bifurcation. The pressure will become higher in a sudden time, and the velocity is lower. So it is the optimal place for vasculopathy to happen.

4. As the bloodstream flows into branch vessels, the speed and pressure will decrease.

\section{Literature References}

[1] Politis A K, Stabropoulos G P, Christolis M N, et al. Numerical modeling of simulated blood flow in idealized composite arterial coronary grafts: steady state simulations [J]. Journal of Biomechanics, 2007, 40(5): 1125-1136.

[2] El-Menyar AA, Al Suwaidi J, Holmes D R. Left main coronary artery stenosis: state-of-the-art [J]. Curr Probl Cardiol, 2007, 32(3): 103-193.

[3] Sianos G, Papafaklis M I, Daemen J, et al. Angiographic sent thrombosis after routine use of drug-eluting stents in ST-segment elevation myocardial infarction: the importance of thrombus burden [J]. Journal of American College of Cardiology, 2007, 50(7): 573-583.

[4] Grube E, Buellesfeld L, Neumann F J, et al. Six-month clinical and angiographic results of a dedicated drug-eluting stent for the treatment of coronary bifurcation narrowings $[\mathrm{J}]$. The American Journal of Cardiology, 2007, 99(12): 1691-1697.

[5] Biondi-Zoccai G G, Sanqiorqi G M, Antoniucci D, et al. Testing prospectively the effectiveness and safety of paclitaxel-eluting stents in over 1000 very high-risk patients: design, baseline characteristics, procedural data and in-hospital outcomes of the multicenter Taxus in Real-life Usage Evaluation Study [J]. International Journal of Cardiology, 2007, 117(3): 349-354.

[6] Shu J, Wang D Q, Jin Y B, et al. A computer simulation study of acute homodynamic changes of biventricular pacing in patients with sick sinus syndrome and its ionic mechanism[J]. Journal of Xi'an Jiaotong University: Medical Sciences,2009, 30( 2): 185-188. ( in Chinese)

[7] Liu Z M, Ma R Y, Liu X. Effection of thrombus to blood flow in uniform arteria straight vein[J]. Journal of Beijing University of Technology, 2008, 34( Supp. ) : 78-82. ( in Chinese) 